\title{
An examination of the interactive video CAPTCHA method to resist relay attack
}

\author{
Ryohei Tatsuda ${ }^{1}$, Kentaro Aburada ${ }^{1 a)}$, Hisaaki Yamaba ${ }^{1}$, \\ Tetsuro Katayama ${ }^{1}$, Masayuki Mukunoki ${ }^{1}$, Mirang Park ${ }^{2}$, \\ and Naonobu Okazaki ${ }^{1}$ \\ ${ }^{1}$ University of Miyazaki, Japan \\ ${ }^{2}$ Kanagawa Institute of Technology, Japan \\ a) aburada@cs.miyazaki-u.ac.jp
}

Abstract: CAPTCHA is a kind of challenge response test, which is used to distinguish human users from malicious computer program such as bots. However, the attack technique called relay attack as a method to avoid the CAPTCHA has been devised. This attack relays the CAPTCHA challenges to remote human-solvers, let them to decode CAPTCHA challenges. We used delay time that is caused by communications needed in relay attack. Our new CAPTCHA uses this delay time between communications to prevent relay attacks. We constructed an experimental environment in which relay attack can be simulated, made a series of experiments in order to evaluate the performance of the proposed method.

Keywords: CAPTCHA, bot, relay attack, human solvers, delay time Classification: Multimedia Systems for Communications

\section{References}

[1] L. von Ahn, M. Blum, N. J. Hopper, and J. Langford, "CAPTCHA: Telling humans and computers apart automatically," Advances in Cryptology, Eurocrypt'03, vol. 2656, Lect. Notes Comput. Sci., pp. 294-311, 2003.

[2] M. Mohamed, S. Gao, N. Saxena, and C. Zhang, "Dynamic cognitive game captcha usability and detection of streaming-based farming," The Workshop on Usable Security (USEC), co-located with NDSS, 2014.

[3] M. Mohamed, N. Sachdeva, M. Georgescu, S. Gao, N. Saxena, C. Zhang, P. Kumaraguru, P. C. van Oorschot, and W.-B. Chen: "A three-way investigation of a game-CAPTCHA: Automated attacks, relay attacks and usability," Proc. 9th ACM Symposium on Information, Computer and Communications Security, pp. 195-206, 2014. DOI:10.1145/2590296.2590298

[4] J. Yan and A. S. El Ahmad, "Usability of CAPTCHAs or usability isuues in CAPTCHA design," Proc. 4th Symposium on Usable Privacy and Security, ACM, pp. 44-52, 2008. DOI:10.1145/1408664.1408671

[5] M. Motoyama, K. Levchenko, C. Kanich, D. McCoy, G. M. Coelker, and S. Savage, "Re:CAPTCHAs-understanding CAPTCHA-solving services in an economiccontext," USENIX Security Symposium, Washington, pp. 1-18, 2010. 


\section{Introduction}

Along with the spread of Web services, everyone can use various services. However, automated programs called bots are cheating on web services. A reverse turing test called CAPTCHA is used to prevent such cheating [1]. CAPTCHA is a test easy for humans to solve but hard for computers.

However, by the sophistication of the attack techniques of the bot, it has come to be able to decipher the CAPTCHA. Therefore, various CAPTCHA schemes have been studied to strengthen the resistance to bots. However, CAPTCHA's security is not enough only with bot tolerance. An attack method called a relay attack may be used as a method to illegally break through CAPTCHA. Existing CAPTCHA is vulnerable to relay attacks. Because it is a human being to decipher CAPTCHA with a relay attack, it is not effective in countermeasures assuming bots.

In this paper, we focus on the delay time that is caused by communications needed in relay attack, and propose CAPTCHA scheme which makes it difficult to resolve CAPTCHA by relay attacks.

\section{Relay attack}

A relay attack relays the CAPTCHA challenges to remote human-solvers, let them to decode CAPTCHA challenges. Then, using the answer of human-solver to break the CAPTCHA. The remote human-solvers include hired humans for compensation and the user on the Internet. The flow of a typical relay attack is shown below.

1. An attacker accesses to the website on which CAPTCHA is presented, and obtains a question image of CAPTCHA.

2. The acquired image is automatically sent to the website that decipher the CAPTCHA by human-wave tactics.

3. In the website above, human beings in exchange for a reward perform the deciphering of CAPTCHA and return the results of the decryption to the attacker.

4. The attacker breaks CAPTCHA using the decryption result obtained.

There is a DCG-CAPTCHA as an existing technique of relay attack measures [2]. In this CAPTCHA, the user has to perform a game-like cognitive task interacting with a series of dynamic images, such as playing a simple object matching game. Because DCG-CAPTCHA is a CAPTCHA of an interactive and dynamic nature, it offers some level of resistance to relay attack due to network latency of the communication required to relay attack (e.g. Transfer CAPTCHA challenge and decoding result to human-solvers). However, DCG-CAPTCHA has been found to be vulnerable to bots [3].

\section{Design and implementation of proposed CAPTCHA}

DCG-CAPTCHA has resistant to relay attacks, but it is vulnerable to bots [3]. So, we propose a CAPTCHA scheme with not only resistance to relay attack but also with resistance to bots.

\subsection{Authentication process}

In this proposed CAPTCHA scheme, the user recognizes the moving object from 


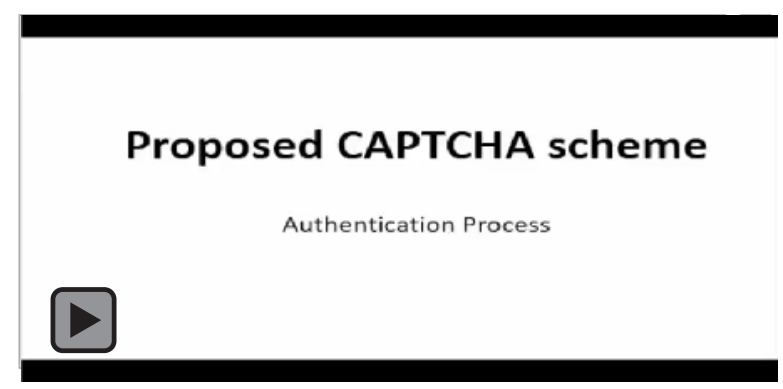

Fig. 1. Proposed CAPTCHA (video).

randomly appearing disturbing objects and tracks the moving object with a mouse cursor (see Fig. 1). (the radius of the object is 25 pixels, the moving object moves about 0.2 7.0 pixels per frame.) The user can solve this CAPTCHA if he/she keeps a mouse cursor pointing on the moving object more than a given time length (e.g. five seconds) during a given trial span (ten seconds in the experiments). We refer to the time that the user keeps a mouse cursor pointing on the moving object as "the tracking success time". (The red gauge on the right side of Fig. 1)

\subsection{Resistance to automated attacks}

In the proposed CAPTCHA scheme, since moving objects and disturbing objects have the same shape, color, and size, it is difficult for bots to detect moving objects from frame images using visual features. For example, you can not track moving objects with a technique that uses patterns of tracked objects such as "template matching" (see Fig. 1).

As an attack against the proposed CAPTCHA scheme, it is conceivable to extract several frames from a video and grasp the position of the moving object using between successive two frames. The proposed CAPTCHA scheme has two features that may become weaknesses. One is that the disturbing object changes its position randomly for each frame. The other is that a running distance of a moving object between consecutive frames is short. Therefore, it is possible to exclude disturbing objects by repeating the AND operation between consecutive frame images (binary images), and it is possible to extract the common area of moving objects. As a result, there is possibility that the position of the moving object may be specified (see Fig. 1).

As a countermeasure to this attack, we changed the design of the object from the initial design. A new object is composed of 12 points, and the points to be displayed are switched for each frame. (Example: display even numbered points, not display odd numbered point) With this countermeasure, it is possible to eliminate the occurrence of the common area of moving objects between consecutive frame images, it will be difficult to specify the position of the moving object even if the logical AND operation is performed (see Fig. 1).

\subsection{Validation for relay attack resistance}

In this study, in order to verify that solution of the proposed CAPTCHA scheme using relay attack becomes difficult due to the delay time, actually we executed relay attack against the proposed scheme CAPTCHA. Relay attack was reproduced 


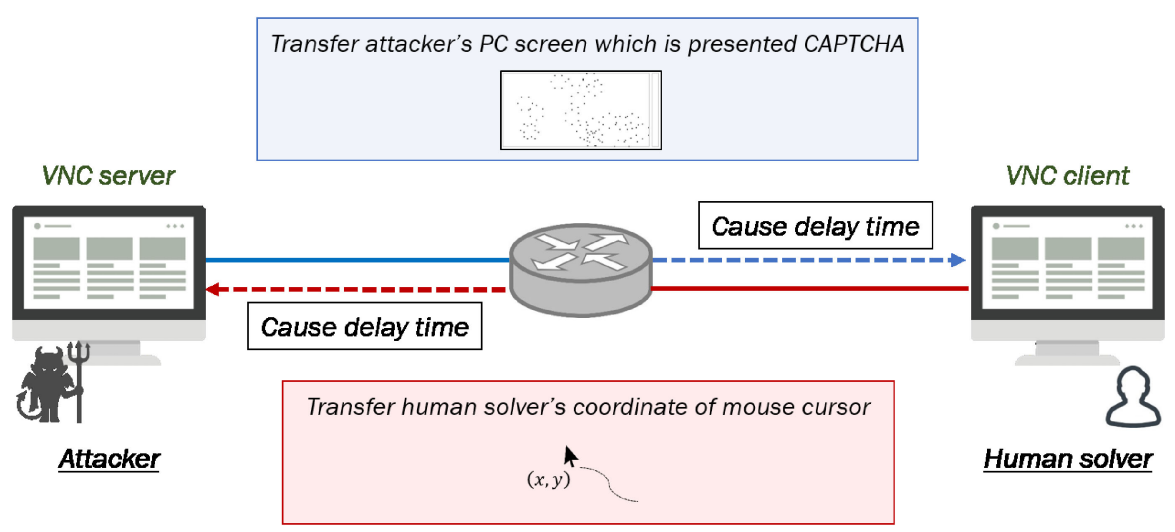

Fig. 2. Relay attack experiment environment.

on a virtual environment using VirtualBox (see Fig. 2). Furthermore, we transfer CAPTCHA challenge using VNC (Virtual Networking Computer) that was used as software for reproducing relay attack in literature [2].

The attacker's PC starts VNC server and displays the proposed CAPTCHA scheme. A human-solver solves the proposed CAPTCHA scheme that is displayed on the screen of the attacker's PC through VNC clients. The length of the delay time of the communication between the attacker's PC and the human-solver's PC will be dependent upon the communication environment. We decided to use VyOS in order to verify relay attack in various communication environments. In this verification experiment, we set the delay time (RTT) of communication between attacker's PC and human-solver's PC to $50 \mathrm{~ms}, 100 \mathrm{~ms}, 200 \mathrm{~ms}$.

10 students of University of Miyazaki participated the experiment. They have solved the proposed CAPTCHA scheme five times with legitimate access and relay attack, we measured "the tracking success time". We run relay attack with 4 patterns communication environment (No insertion of delay time, RTT $=50 \mathrm{~ms}$, $100 \mathrm{~ms}, 200 \mathrm{~ms})$.

\subsubsection{Experimental results}

Fig. 3 shows the histograms of the tracking success time when ten experiment participants solved the proposed CAPTCHA scheme. From the experimental results, we confirmed that tracking of moving object is more difficult as the delay time of a relay attack increases.

We determine the threshold of the tracking success time which is difficult for the human solver to achieve from the data of successful tracking time obtained in the experiment. The normality of distribution of successful tracking time data sets were checked using the Shapiro-Wilk's test. We conducted a test at a significance level of 5\%. As a result, we found that the data of successful tracking time of the legitimate access and the data of successful tracking time of the relay attack with the delay time of $50 \mathrm{~ms}$ follow the normal distribution. We approximated data of successful tracking time of legitimate access and relay attack $(R T T=50 \mathrm{~ms})$ to a normal distribution (show Fig. 3(e)). We calculated RAR (Relay attack Acceptance Rate) and $L R R$ (Legitimate user Rejection Rate) from a normal distribution (show Fig. 3(f)). We investigate a threshold value of successful tracking time for preventing a relay attack based on RAR and LRR. 


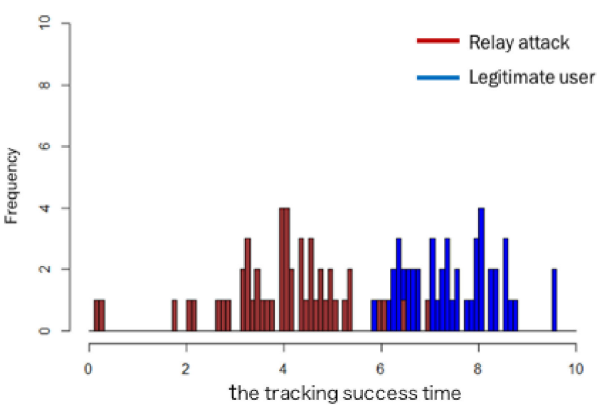

(a) No insertion of delay time

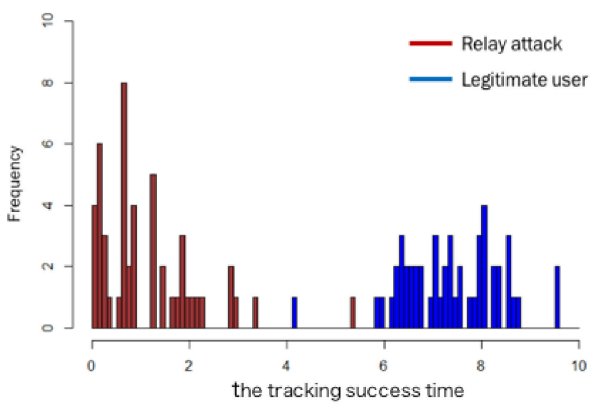

(c) Insert delay time of $100 \mathrm{~ms}$

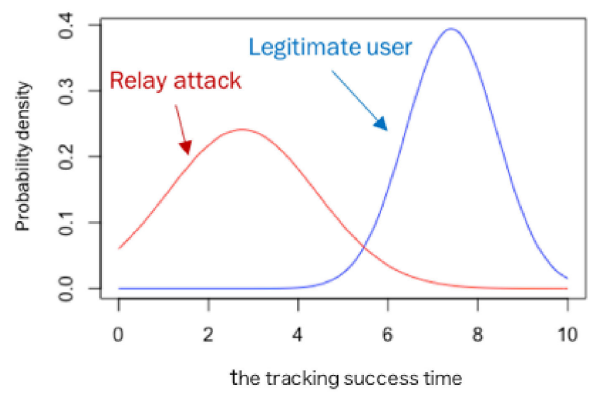

(e) Normal distribution of successful tracking time (RTT=50ms)

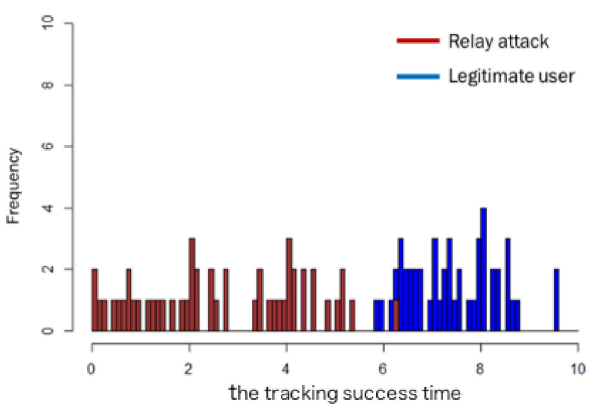

(b) Insert delay time of $50 \mathrm{~ms}$

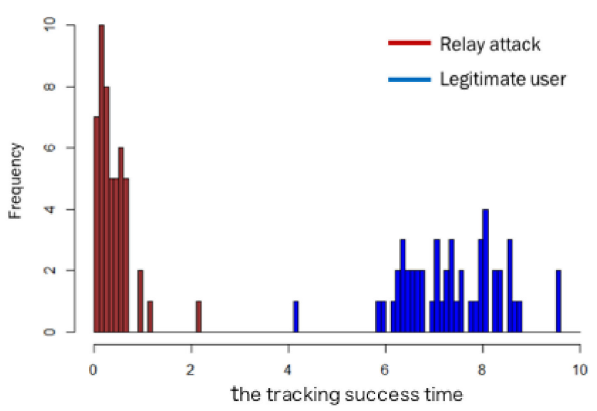

(d) Insert delay time of $200 \mathrm{~ms}$

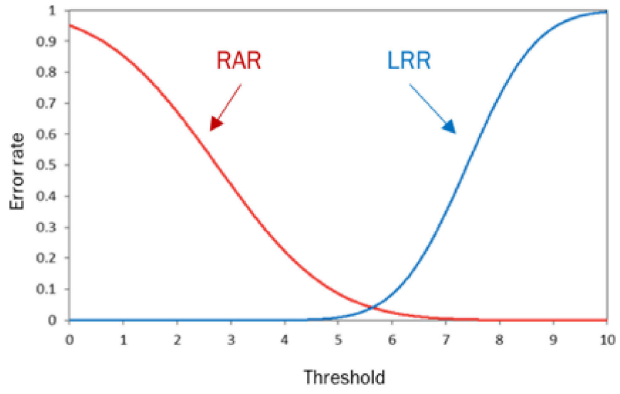

(f) RAR (Relay attack Acceptance Rate) and LRR (Legitimate user Rejection Rate)

Fig. 3. Experiment results.

According to literature [4], the average success rate of reCAPTCHA which are commonly used us about $97 \%$. If the proposed scheme CAPTCHA wants to have a success rate of comparable legitimate user, set LRR to 3\%. When LRR is 3\%, threshold of successful tracking time is about 5.5 seconds. Also, when setting the threshold value to 5.5 seconds, RAR is $5 \%$. At this time, if relay attack is performed in communication environment $50 \mathrm{~ms}$ delay occurs, it is possible to prevent about $95 \%$ of relay attack. According to the literature [5], the human solver is getting a reward of $\$ 0.5$ to $\$ 3.0$ per 1000 CAPTCHA pieces of decoding. If RAR is $5 \%$, the relay attack will not be economically feasible because even if 1000 CAPTCHAs are solved, about 50 of them will be success.

\section{Conclusion}

As a method to avoid the CAPTCHA, there is an attack method called relay attack is used. Conventional countermeasures assuming bots of CAPTCHA have no effect on relay attack. In this paper, we use the delay time due to the communication 
required to relay attack and proposed a CAPTCHA scheme that makes it difficult to answer in the relay attack. We performed a relay attack against the proposed scheme CAPTCHA to verify relay attack resistant. As a result, the proposed method CAPTCHA showed that it is possible to have a resistance to relay attack.

As a future task, we need to investigate attack methods based on other image processing. In addition, we should evaluate the usability of the proposed CAPTCHA.

\section{Acknowledgments}

This work was supported by JSPS KAKENHI Grant Numbers JP17H01736, JP17K00139. 